\title{
El tiempo productivo, reproductivo y de ocio en las parejas de doble ingreso*
}

\author{
Marc Ajenjo Cosp \\ Universitat Autònoma de Barcelona. Departament de Sociologia \\ marc.ajenjo@uab.cat \\ Joan García Román \\ Universitat Autònoma de Barcelona. Centre d'Estudis Demogràfics \\ jgarcia@ced.uab.cat
}

Recibido: 08-05-2010

Aceptado: 26-04-2011

\section{Resumen}

A pesar de que la incorporación de la mujer al mundo laboral rompe con el principio más elemental del male breadwinner model, algunas de sus características siguen vigentes. Para abordar con más detalle esta vigencia, y a partir de la Encuesta de Empleo del Tiempo 2002-2003, el artículo compara el reparto del tiempo cotidiano que tiene lugar en las parejas de doble ingreso y el que tiene lugar en las parejas donde sólo trabaja el varón.

Esta comparación permite concluir que, a pesar de que, en las parejas de doble ingreso, los hombres ya no son los únicos breadwinner ni las mujeres las únicas cuidadoras, siguen siendo los principales. Además, la aportación respectiva de unos y otras al trabajo no remunerado y remunerado es muy desigual, una desigualdad que comporta una fuerte diferencia en el tiempo de ocio.

Solamente las parejas de doble ingreso de características muy concretas han conseguido cierto grado de equidad: parejas jóvenes, cohabitantes, sin hijos y que el trabajo de la mujer le aporta cierto poder adquisitivo. El simple cambio en alguna de estas categorías comporta un elevado grado de desigualdad, y es la presencia de hijos en el hogar la característica más determinante. En el caso concreto de la edad, se destaca que las parejas jóvenes son más igualitarias, no por pertenecer a una generación que acepta más fácilmente la igualdad, sino por las características asociadas a la edad, una conclusión nada esperanzadora de cara a una mayor equidad futura entre hombres y mujeres.

Palabras clave: parejas de doble ingreso; género; uso del tiempo.

Abstract. Productive time, reproductive time and leisure time amongst dual-earner couples

Although increased female labour force participation changed the dominant male breadwinner model, some of its characteristics are still firmly in place. Using data from the Time

* Este trabajo se inscribe dentro del proyecto El reparto del tiempo y el uso del espacio en las parejas biactivas. La situación española en el contexto europeo (Ref. CSO2009-08273 I GEOG) subvencionado por el plan nacional I+D+I del Ministerio de Educación y Ciencia; además de formar parte de la tesis doctoral de Joan García Román, inscrita en el doctorado de Demografía de la Universitat Autònoma de Barcelona. 
Use Survey 2002-2003, this article compares the day-to-day sharing of time in dual-earner couples and couples where only men are employed. This comparison suggests that, despite the fact that in dual-earner couples breadwinning and care-giving activities are shared, males are still generally primary earners and women the primary care providers. In addition, we find the contribution to unpaid and paid work of men and women is not equal, and this inequality also presupposes a strong disparity in leisure time. Only dual-earner couples with specific characteristics have achieved a certain degree of fairness in the sharing of responsibilities, namely young, cohabiting couples with no children, where the female partner contributes a certain level of earnings. A change in those variables leads to a higher level of inequality, with the presence of children having the most impact. In relation to age, we find that young couples are more egualitarian, not because this generation more readily accepts a norm of gender equality but because of certain characteristics related to age. Our findings offer little hope for a future with more equality between men and women.

Key words: dual-earner couples; male breadwinner model; gender; time-use.

\section{Sumario}

\section{Introducción}

2. Antecedentes y estado actual de los conocimientos científico-técnicos

3. Objetivos e hipótesis de partida

4. Fuente utilizada y metodología

5. El uso del tiempo en las parejas tradicionales y en las parejas

de doble ingreso
6. Los condicionantes de las diferencias por género de empleo del tiempo en las parejas de doble ingreso

7. Conclusiones

Bibliografía

\section{Introducción}

El objeto de estudio de este artículo son las parejas cuyos dos miembros están ocupados, aquellas que se denominan habitualmente "parejas de doble ingreso» (del inglés dual-earner couples). En concreto, nos proponemos analizar el reparto del tiempo cotidiano en estas parejas, un aspecto de la realidad social estrechamente vinculado con el bienestar de las personas, un buen indicador de las relaciones sociales y un buen termómetro de las desigualdades entre hombres y mujeres y de las relaciones de poder en el seno de las parejas (Perulli, 1996; Moreno, 2007). Además, el análisis del tiempo ha contribuido, en términos generales, a la visibilización del trabajo doméstico, un trabajo que, desde un punto de vista económico y social, ha sido poco valorado (Durán, 1986).

En España, tal y como sucede en el resto de Europa, las parejas de doble ingreso aumentan considerablemente desde la década de 1990, cuando pasan de representar el 31\% en 1992 al 43\% en tan solo ocho años (Franco y Winqvist, 2002). Se trata, sin duda, de un importante incremento, y más teniendo en cuenta que es entre las parejas de nueva formación donde mayor es su incidencia.

El principal motor de este incremento es la incorporación al mercado laboral de las mujeres, una incorporación que en España se encuentra claramente 
en alza, a pesar de que, tal y como sucede en otros países del sur, es más tardía que en el resto del continente (Alberdi, 1999). Este retraso tiene un componente claramente generacional, de modo que, a medio plazo, es previsible un incremento de la actividad femenina hasta valores similares a los encontrados en otros países europeos (Tobío, 2001). A pesar de esta previsión, no debemos olvidar que la crisis económica actual, con el incremento consiguiente de la tasa de paro, incidirá en la "normalidad estadística» de estas parejas.

Hoy en día, esta «normalidad» tiene dos caras que difieren y se complementan al mismo tiempo. Por un lado, el conjunto de la sociedad ha asumido la participación femenina en el mercado laboral, lo cual ha convertido a las parejas de doble ingreso en la forma de convivencia mejor valorada (Dema, 2005; MacInnes, 2005); mientras que, por el otro, sigue vigente el modelo de cabeza de familia masculino, de modo que, a pesar de la creciente visibilidad de las mujeres en el mercado de trabajo, socialmente se les sigue atribuyendo el papel principal de esposa, madre e hija (Moreno, 2007). A modo de ejemplo, a pesar de aceptarse la presencia femenina en el mercado laboral, no por ello existe una preocupación social por su paro, una preocupación que sí merece el paro masculino (Torns, 2001).

En los últimos años, se ha producido en Europa un incremento de la diversificación de los tiempos de trabajo (Gasparini et al., 2000), que ha sido posible gracias a los nuevos métodos de producción y formas de organización y, sobre todo, a la mayor participación de la mujer en el mercado laboral (Fagan, 2003). Si bien el trabajo a tiempo parcial ha sido publicitado como una forma de promover la flexibilidad en el mercado laboral de acuerdo con la política de protección familiar y la redistribución de la ocupación, en España, tal y como sucede en otros países del sur como Italia, Grecia o Portugal, se sigue dando una escasa presencia de lugares de trabajo a tiempo parcial, una forma de ocupación que es rechazada por la mayoría de trabajadores españoles (Brullet, 2000; Tobío, 2001; Parella; 2003; Fagan, 2003; Torns y Miguélez, 2007). Ahora bien, y a pesar de que en otros países europeos, como en Holanda, la presencia de trabajo a tiempo parcial es mucho más común, en parte porque no se trata de una oportunidad empresarial para la reducción de los gastos, sino que sus características son similares al trabajo a tiempo completo (Méda, 2002; Borràs et al., 2007), la fuerte presencia de mujeres en estos trabajos tampoco es síntoma de igualdad de género (Plantenga et al., 1999).

Esta característica de la ocupación tiene, sin duda, una fuerte incidencia en los usos del tiempo, sobre todo en aquellas parejas donde los dos miembros trabajan. La comparación del sistema de actividades y el uso del tiempo en función del género muestra que las mujeres dedican mucho más tiempo que los hombres a las labores del hogar, lo cual genera un sistema desigual de uso del tiempo. En España, existen distintos estudios que facilitan información sobre el menor tiempo que las mujeres disponen para ellas en su vida cotidiana en comparación con los hombres. Dichos estudios introducen la dimensión temporal para conocer mejor la organización de la sociedad y las desigualdades que le son inherentes (Durán, 1986; Izquierdo et al., 1988; García Ramón, 
1989; Ramos, 1990; Sabaté et al., 1995; Álvaro, 1996; Valiente, 1997; Raldúa, 2001; García Díez, 2003; Larrañaga et al., 2004; Moreno, 2007, Callejo et al., 2008; González y Jurado, 2009; entre otros).

\section{Antecedentes y estado actual de los conocimientos científico-técnicos}

Generalmente, la diferenciación sexual de las actividades y el uso del tiempo es analizada como producto de una más o menos desarrollada división del trabajo. Desde la teoría económica neoclásica, se incide en el concepto de máxima eficiencia de la unidad familiar para explicar la especialización de las tareas (Becker, 1981). Para la teoría del rol social, en cambio, las distintas conductas sociales de mujeres y hombres son consecuencia de los roles que se les atribuyen, una diferencia que tiene su origen en la división sexual del trabajo (Eagly, 1983, 1987).

El reparto del tiempo en el seno de las parejas debe analizarse en función del sistema de relaciones que se establecen en el interior de las familias, unas relaciones que, como todas las relaciones de poder, generan conflictos (Dema, 2006). Ahora bien, este poder no se limita a aspectos materiales, sino que también devienen importantes factores ideológicos y culturales de la propia pareja (Ibáñez, 2008), las relaciones sociales u otros factores externos, como puede ser el poder que otorga el Estado con sus intervenciones (Brullet, 2000; Borràs et al., 2007). Entre el conjunto de aspectos a considerar, adquieren una relevancia especial las normas sociales y los roles asociados tradicionalmente a hombres y mujeres, que tienen, sin duda, un peso importante en las relaciones de pareja en general y en el uso del tiempo en particular. En este sentido, el simbolismo de la masculinidad y la feminidad incide fuertemente en los comportamientos individuales, de modo que su pérdida en algún aspecto suele ser compensada en otros; tal y como sucede en las parejas donde los ingresos de la mujer superan a los del hombre, las cuales reproducen una división más tradicional del trabajo doméstico (Bittman et al., 2003).

Y es que el cambio de roles no es aceptado fácilmente, sobre todo por parte de los hombres. La situación en que la mujer gana más que el hombre y éste se ocupa mayoritariamente de las tareas domésticas, genera muchas reticencias en el resto de familia y allegados (Brines, 1994; Tobío, 2001). La percepción social sigue siendo que el trabajo remunerado es opcional para las mujeres, pero no así las «obligaciones» domésticas (Lewis, 2001; Parella y Samper, 2007), mientras que para el hombre la situación es exactamente la opuesta, algo que sin duda incide en los tiempos que unos y otras dedican a ambas tareas.

No debemos olvidar, además, que la incorporación masiva de la mujer al mundo laboral esconde, en el fondo, unas notables diferencias con la actividad masculina: se ha concentrado en ocupaciones con contenidos próximos a la definición tradicional de tareas femeninas, mientras que ha sido excluida de los lugares de mando y responsabilidad y, además, la contraprestación salarial es más baja (Valiente, 1997; Beck y Beck-Gernsheim, 1998; Bourdieu, 2000; Tobío, 2001; Méda, 2002; Dema, 2006; Mercadé, 2007). Además, la diferencia 
es notable en otros aspectos, ya que, a pesar de incrementar la proporción de activas, también lo hace su desocupación (Beck y Beck-Gernsheim, 1998). Además, en el momento en que una actividad se feminiza, pierde valor. En consecuencia, los hombres siguen dominando el espacio público, el campo de poder, mientras que las mujeres continúan dedicándose, primordialmente, al espacio privado o a distintas extensiones de este espacio, como son los servicios sociales y educativos o los universos de producción simbólica (Bourdieu, 2000).

Las teorías de la individualización y de la modernidad reflexiva apuntan que los cambios acaecidos en el interior de las familias conllevan la coexistencia de distintos tipos de familia. Las familias contemporáneas se fundamentan en el individuo y en su desarrollo, de modo que son los individuos los rectores de su propia forma de vida. La familia, el matrimonio, la paternidad, la sexualidad, el erotismo y el amor ya no pueden ser presupuestos ni establecidos de forma obligatoria, sino que deben ser descifrados, negociados, acordados y fundamentados en todos sus detalles (Beck y Beck-Gernsheim, 1998). Existe, pues, una gran diferencia entre el matrimonio tradicional y el actual, basado en lo que Giddens (1998) denomina «ideales de la pura relación», que se sustentan en la implicación de cada individuo en las condiciones de la asociación, en lo que sería la democracia en la esfera privada. Ahora bien, estas teorías no intentan explicar las diferencias de poder en el interior de la pareja, sino que plantean su superación (Ibáñez, 2008), en tanto que analizan las relaciones de pareja en términos de relaciones entre iguales; olvidando, por un lado, la fuerte carga de las normas sociales y, por el otro, que la individualización no tiene el mismo significado para hombres que para mujeres (Dema, 2006). Y es que la fuerza del orden establecido está fundamentada en un sistema social y económico centrado en la producción, sin otorgar apenas valor alguno a la reproducción de los individuos, a pesar de que es la garante del bienestar material y emocional de las personas, así como de la existencia del sistema productivo (Parella y Samper, 2007). Aunque, a priori, no está establecido quién friega los platos y cuándo, quién cambia los pañales a los bebés, quién compra ni quién pasa el aspirador, quién gana el pan..., la toma de estas decisiones por parte de las parejas muestra la existencia de fuertes desigualdades.

La mayor parte de estas cuestiones conducen a preguntarnos cuál es la situación actual en relación con el male breadwinner model $l^{1}$, una cuestión que ha sido planteada por un buen número de investigaciones y que a menudo viene de la mano de la reflexión sobre el Estado del bienestar. Así, la tipología propuesta por Esping-Andersen (1990), basada en el concepto de desmercantilización y que define tres regímenes de bienestar - el corporativo-conservador, el liberal y el socialdemócrata—, es criticada desde una perspectiva del género porque centra su interés exclusivamente en el mercado laboral, olvidando la importancia que tiene el trabajo no remunerado de la mujer en el hogar para la misma reproducción de la fuerza de trabajo (Lewis, 1992; Orloff, 1993).

1. De ahora en adelante, para designar el concepto de male breadwinner model se va a utilizar directamente su traducción: modelo tradicional. 
Si bien el incremento de la actividad femenina conduce a algunas autoras a aventurar el declive del modelo tradicional (Lewis, 2001; Orloff, 2006), los cambios acaecidos en la estructura familiar no son tan evidentes, sino que persiste la división de género dentro del hogar y sigue intacta la figura del cabeza de familia. Así, algunos de los principios del modelo apenas han sufrido alteraciones (Brines, 1994; Hakim, 2000; Moreno, 2007), sobre todo aquéllos que se refieren a la incorporación de los hombres al trabajo no remunerado (Lewis, 2001; Méda, 2002; MacInnes, 2005).

Volviendo al estado de salud del modelo tradicional, cabe destacar, en primer lugar, que, en sentido estricto, se trata de un modelo que nunca ha existido, en cuanto que las mujeres, excepto en algunos periodos, países y clases sociales concretas, siempre han trabajado en la producción (Lewis, 1992; Durán, 2007). En segundo lugar, y como ya hemos destacado, la mayoría de investigaciones coinciden que, en relación con la incorporación de la mujer al trabajo remunerado, se trata de un modelo en declive, el cual tiene sus particularidades territoriales y sociales. Como describe Lewis (2001), se está produciendo un cambio pendular hacia un modelo donde la actividad laboral, ya sea de hombres y mujeres, deviene prioritaria sobre la actividad reproductiva: nos encontramos en un periodo de transición entre el male breadwinner y el adult worker, donde hombres y mujeres trabajan en igualdad de condiciones. En concreto, la autora británica describe hasta seis modelos combinando la dedicación de hombres y de mujeres al trabajo remunerado (tiempo parcial y tiempo completo) y las distintas opciones de cuidado que se establecen en el seno del hogar (la mujer, el hombre, el mercado, la familia, el Estado, etc.).

\section{Objetivos e hipótesis de partida}

El principal objetivo de este artículo es evaluar la persistencia de algunos de los principios básicos sobre los que se fundamenta el modelo tradicional en España, y hacerlo en las parejas de doble ingreso, que, por definición, rompen con el principio más elemental de este modelo: dejar en manos del hombre las tareas productivas. A pesar de que la masiva incorporación de la mujer al mundo laboral sacude uno de los pilares del modelo, no tiene por qué alterar otros aspectos que le son consustanciales, como es el desigual reparto de las tareas del hogar.

Este desigual reparto se evidencia al analizar el tiempo que hombres y mujeres dedican a estas tareas. Según la Encuesta de Empleo del Tiempo 20022003, las mujeres de 20 a 74 años dedican al trabajo doméstico-familiar unas 3 horas 18 minutos diarios más que los hombres; una diferencia que, junto con la que se produce en Italia, es de las más elevadas de la Unión Europea y que contrasta enormemente con la que se produce en los países nórdicos (Aliaga, 2006). Una conclusión que no dista demasiado de la que han llegado otras investigaciones realizadas con metodologías más o menos parecidas (Izquierdo et al., 1988; Ramos, 1990; Álvaro, 1996; Valiente, 1997; Raldúa, 2001; García Díez, 2003; Larrañaga et al., 2004; MacInnes, 2005; Balcells, 2009). 
Ahora bien, también es verdad que en España, tal y como sucede en Italia, es donde menor es la presencia de parejas de doble ingreso, y que el cálculo del reparto del tiempo para el conjunto de hombres y mujeres viene mediatizado por esta menor presencia.

Así, la mayoría de investigaciones analizan el reparto del tiempo entre hombres y mujeres para el conjunto de la población, o bien centrándose en hombres y mujeres que tienen una ocupación. A pesar de la validez de estas comparaciones, no son estrictamente entre iguales, ya que un buen número de hombres ocupados en el mercado laboral conviven con mujeres que no están ocupadas, mientras que la situación contraria no es muy frecuente. Es por este motivo que consideramos interesante que, para comprender mejor el desigual reparto del tiempo entre sexos, sea de cabal importancia el análisis de hombres y mujeres de características laborables y familiares similares, característica que cumplen las parejas de doble ingreso. En España, un análisis parecido lo han desarrollado González y Jurado (2009), aunque, en su caso, está limitado a lo que han llamado las "tareas domésticas rutinarias», que excluye el cuidado a niños u otros dependientes, las tareas de relaciones sociales y de cuidados emocionales, las tareas de organización mental y las más esporádicas, como la jardinería o las reparaciones.

Una de las preguntas que planteamos en nuestro artículo es hasta qué punto las parejas de doble ingreso han emprendido la transición hacia un nuevo modelo. La hipótesis de partida es que los rasgos más esenciales del viejo modelo siguen vigentes, y que la equiparación de roles entre hombres y mujeres dista mucho de alcanzarse: a pesar de cierta igualdad en los tiempos productivos, se observarán diferencias significativas en el tiempo destinado al trabajo no remunerado. En este sentido, es probable que estas parejas se sitúen en uno de los primeros modelos dual-breadwinner propuestos por Lewis (2001), caracterizados por un tiempo productivo que, a pesar de cierta equiparación, dista de una total igualdad y un tiempo reproductivo muy desigualUn segundo objetivo se refiere a las variables sociodemográficas que más inciden en un reparto más igualitario del tiempo entre los hombres y las mujeres de una pareja de doble ingreso. En este sentido, la mayoría de investigaciones realizadas para el conjunto de la población muestran que estas variables son, además de la situación laboral de la mujer, la edad (o generación), el nivel educativo, la cohabitación, el ciclo de vida, el volumen de ingresos, la categoría socioeconómica, la homogamia, el tipo de jornada laboral, la externalización de una parte del trabajo doméstico y la religiosidad, entre otras (Izquierdo et al., 1988; Ramos, 1990; Gershuny y Sullivan, 1998; Méda, 2002; García Díez, 2003; Larrañaga et al., 2004; MacInnes, 2005; Callejo et al., 2008; Balcells, 2009; González y Jurado, 2009).

Aquí nos planteamos esta cuestión solamente para las parejas de doble ingreso, identificando las variables más explicativas de la igualdad de reparto del tiempo. Si se tienen en cuenta solamente las tareas más rutinarias, las variables más predictivas son los ingresos de la mujer, la cohabitación y la relación que tiene el hombre con el mercado laboral (González y Jurado, 2009). 


\section{Fuente utilizada y metodología}

Para analizar parejas de doble ingreso, se requiere de una fuente que interrogue a los dos miembros de una misma pareja. Este requisito lo cumple la Encuesta de Empleo del Tiempo 2002-2003, que, bajo las directrices de Eurostat, se realizó en diferentes países europeos ${ }^{2}$ siguiendo la metodología de los presupuestos del tiempo.

Para España, el tamaño de la muestra es de 20.603 hogares, los cuales contienen información sobre 46.774 individuos mayores de 10 años. Para el análisis, se han seleccionado aquellos hogares donde conviven parejas formadas por personas de distinto sexo, que son un total de 12.756. Además, y como el objetivo es el análisis de parejas de características sociodemográficas similares, y que uno de los ejes principales de esta similitud es la actividad laboral, se han excluido todas aquellas donde alguno de sus miembros se encuentra en edad de jubilación; de modo que el tamaño de la muestra se ha reducido a 9.487 parejas.

Con el fin de evaluar el uso que hombres y mujeres hacen de su tiempo, se ha comparado, para cada pareja, el tiempo que él y ella dedican a cada una de las actividades. Se ha calculado un indicador específico, que consiste en la relación entre la diferencia absoluta de horas y el total de horas que ambos miembros dedican a cada una de las tareas, y se ha denominado «indicador de desigualdad $»^{3}$.

Para acotar qué variables inciden en un reparto más igualitario entre mujeres y hombres integrantes de una pareja de doble ingreso, se han desarrollado modelos distintos de análisis de la varianza de efectos fijos. A pesar de que el objetivo principal es analizar qué categorías de las variables independientes comportan una mayor o menor diferencia en el tiempo empleado por parte de mujeres y hombres al trabajo no remunerado, se ha considerado que esta diferencia no podía desvincularse de las diferencias observadas en los tiempos destinados a trabajo remunerado y a ocio ${ }^{4}$. Al estar las tres variables

2. Se puede obtener información más detallada de la fuente, así como de algunos resultados relevantes en Aliaga y Winqvist (2003) y en Aliaga (2006), o bien accediendo a la pàgina web http://www.ine.es/jaxi/menu.do?type=pcaxis \&path=\%2Ft25\%2Fe447\&file=inebase $\& \mathrm{~L}=0$.

3. La ventaja principal de este indicador es que toma valores comprendidos entre -1 y 1 , donde cero es sinónimo de plena igualdad, mientras que un valor próximo a -1 comporta que sea el hombre el que se encargue por completo de aquella actividad, y un valor próximo a 1 se corresponde a la situación inversa.

$\mathrm{Si}$ llamamos $\mathrm{H}$ al tiempo empleado por el hombre a una tarea y $\mathrm{M}$ al tiempo empleado por la mujer, el indicador debería escribirse como

$$
\text { IDes }=\frac{M-H}{M+H}
$$

4. Para acotar el tiempo destinado a trabajo remunerado, se ha considerado, además de la actividad laboral propiamente dicha, las actividades y los desplazamientos relacionados con ella.

En el tiempo de trabajo no remunerado, se han incluido los tiempos dedicados al hogar - cocina, limpieza, ropa, jardinería, animales de compañía, reparaciones, compras, gestiones, etc.—, al cuidado de la familia — niños y adultos— y a los desplazamientos relacionados. 
relacionadas entre sí, se ha optado por la técnica del análisis multivariante de la varianza (MANOVA), utilizando las diferencias entre la mujer y el hombre en cada uno de esos tres tiempos. El MANOVA no es más que una extensión del análisis univariante de la varianza, donde se calcula un único valor estadístico para el contraste de la igualdad de medias de las tres variables dependientes. Para ello, se generan $k$ vectores de medias a partir de la combinación de las categorías de las variables independientes cuyos componentes son las medias de las variables dependientes (la diferencia de tiempo en los tres tipos de tareas).

$$
\vec{\mu}_{\mathrm{i}}=\left(\mu_{\text {dif_tareas_prod }}, \mu_{\text {dif_tarea__reprod }}, \mu_{\text {dif_tareas_ocio }}\right)
$$

MANOVA contrasta la existencia de diferencias entre estos vectores:

$$
\left\{\begin{array}{l}
H_{0}: \vec{\mu}_{1}=\vec{\mu}_{2}=\ldots=\vec{\mu}_{\mathrm{k}} \\
H_{1}: \text { algún vector distinto }
\end{array}\right.
$$

Se han realizado tipos de análisis multivariante distintos, utilizando siempre como variables dependientes las diferencias entre mujeres y hombres en el uso del tiempo para las tres tareas citadas. En primer lugar, modelos en los que se han incorporado las distintas variables independientes de forma separada (véanse los resultados en las columnas «Modelo de factor único» en el cuadro 2). En segundo lugar, y con el fin de evaluar la contribución de cada variable eliminando el efecto de las restantes, se ha incorporado el conjunto de variables al mismo tiempo (véanse los resultados en las columnas «Modelo multivariante» en el cuadro 2).

Las tablas muestran los coeficientes estimados por el modelo, los cuales se interpretan del mismo modo que los coeficientes obtenidos en una regresión que utilice variables categóricas, ya que este análisis no es más que un caso específico de los modelos lineales generales.

\section{El uso del tiempo en las parejas tradicionales $\mathrm{y}$ en las parejas de doble ingreso}

En función de la relación que ambos miembros de la pareja mantienen con la ocupación, podemos dividir las parejas en cuatro grupos: no trabaja ningún miembro, sólo trabaja la mujer, sólo trabaja el hombre y parejas de doble ingreso. Según la Encuesta de Empleo del Tiempo 2002-2003, entre las parejas cuyos ambos miembros tienen menos de 65 años, dos son los tipos más numerosos: las parejas en las que sólo trabaja el hombre, que representan un $43,1 \%$ de este total, y las parejas de doble ingreso, que suponen un $40,4 \% \mathrm{del}$

Por otro lado, el concepto de ocio utilizado incluye la vida social, la diversión, el ocio pasivo, los deportes y las actividades al aire libre, las aficiones, los juegos, los medios de comunicación, el voluntariado y los trayectos asociados a alguno de estos motivos. 
total; mientras que en un $11,5 \%$ de parejas no trabaja ningún miembro y en un $5,0 \%$ solamente trabaja la mujer ${ }^{5}$.

Observamos, en primer lugar, que las parejas de doble ingreso son significativamente más jóvenes que el resto, una juventud que debe ser explicada tanto en términos de generación como de edad. En términos de generación, porque la incorporación al mercado laboral de las generaciones más recientes se ha llevado a cabo en un momento donde la incorporación de la mujer era mucho más importante que unos años atrás (Tobío, 2001). Y en términos de edad, por el efecto de las prejubilaciones en un cambio de tipología, de modo que una pareja en la que ambos miembros han trabajado durante toda su vida laboral tiene una elevada probabilidad de convertirse en uniactiva al acercarse a la edad de la jubilación. Es por este motivo que, en las parejas en las cuales no trabaja ninguno de los dos miembros, la edad es más elevada que en el resto. Algo parecido sucede con las parejas en las que sólo trabaja la mujer, que no se caracterizan por haber invertido los roles tradicionales de hombre productor y mujer reproductora, una situación que, en términos generales, es muy poco común (Brines, 1994; Tobío, 2001; Lewis, 2001): en solamente un 4,3\% de estas parejas, los hombres declaran estar realizando labores del hogar como actividad principal, mientras que la mayoría declaran estar en paro o temporalmente ausentes de la ocupación.

Mucho menores son las diferencias de edad entre las parejas en las cuales sólo trabajan los hombres y las parejas de doble ingreso. Las primeras constituyen el modelo más tradicional de pareja, con los roles completamente compartimentados: prácticamente el $80 \%$ de estas mujeres declara que su relación con la actividad es la de "ama de casa». Y estos son los dos tipos de parejas en las que se va analizar el uso del tiempo: aquellas en las que solamente trabaja el hombre, prototipo del modelo tradicional, y las parejas de doble ingreso, prototipo de los nuevos modelos de pareja.

Una mirada rápida al uso del tiempo de unas parejas y otras revela que, independientemente del sexo, las actividades que consumen un mayor número de horas son las que se han clasificado bajo el epígrafe de «cuidados personales» (véase cuadro 1): prácticamente la mitad del día se emplea en el descanso, en la alimentación y en otros aspectos relacionados. Le siguen tres tipos de actividad que son las que se van a analizar más detalladamente y que, en su conjunto, tienen un consumo de tiempo más o menos parecido: las relacionadas con el trabajo remunerado, con el trabajo no remunerado y con el ocio y el tiempo libre.

En lo que respecta al trabajo remunerado, en conjunto, las parejas de doble ingreso dedican a cuestiones laborales un $70 \%$ más de tiempo que las

5. Debemos tener en cuenta que no se trata de grupos cerrados, sino que, a lo largo del ciclo de vida, las parejas pueden cambiar de un grupo a otro: el paro de alguno de sus miembros, una baja por enfermedad, una jubilación o una prejubilación y, sobre todo, una baja por maternidad o paternidad son aspectos que comportan una variación de tipología. Los datos de la encuesta no son más que una foto fija de los modelos de pareja en un momento concreto y no es posible saber hasta qué punto se han dado (y si se van a dar) cambios en la relación con la ocupación. 
Cuadro 1. Diferencia entre hombres y mujeres en el tiempo diario empleado en distintas actividades. Media en horas y minutos e índice de desigualdad. Parejas de doble ingreso y tradicionales menores de 65 años

\begin{tabular}{lcccccc}
\hline & \multicolumn{3}{c}{ Parejas tradicionales } & \multicolumn{2}{c}{ Parejas de doble ingreso } \\
Actividades & \multicolumn{2}{c}{ Horas diarias } & Índice de & \multicolumn{2}{c}{ Horas diarias } & Índice de \\
relacionadas con... & Hombre & Mujer & desigualdad & Hombre & Mujer & desigualdad \\
\hline cuidados personales & $10: 55$ & $11: 10$ & 0,01 & $10: 42$ & $10: 36$ & 0,00 \\
trabajo productivo & $7: 04$ & $0: 07$ & $-0,97$ & $6: 58$ & $5: 21$ & $-0,13$ \\
estudios & $0: 03$ & $0: 08$ & 0,46 & $0: 05$ & $0: 06$ & 0,13 \\
trabajo reproductivo & $1: 30$ & $7: 42$ & 0,67 & $2: 03$ & $4: 31$ & 0,38 \\
ocio, tiempo libre & $4: 26$ & $4: 50$ & 0,04 & $4: 11$ & $3: 23$ & $-0,11$ \\
otros aspectos & $0: 03$ & $0: 03$ & 0,02 & $0: 03$ & $0: 03$ & 0,08 \\
\hline
\end{tabular}

Fuente: elaboración propia a partir de la Encuesta de Empleo del Tiempo 2002-2003.

parejas tradicionales. En contrapartida, las parejas de doble ingreso destinan un 30\% menos de tiempo al trabajo no remunerado, diferencia que se acentúa los días laborables. Esta menor dedicación está directamente relacionada con la externalización de una parte de estas tareas (Tobío, 2001), externalización que se complementa con una mayor dedicación los fines de semana. Centrándonos en el tipo de tarea que las parejas de doble ingreso eluden, observamos que son los aspectos relacionados con la ropa - planchar, sobre todo, pero también lavar- los que, en términos relativos, más les diferencian de las parejas tradicionales; pero también aquellos aspectos relacionados con la limpieza, ya sea de la vajilla o del hogar en su conjunto. En cambio, dedican un tiempo parecido a las parejas tradicionales en las tareas relacionadas con la compra y con los niños, en las que consumen un $20 \%$ menos de tiempo que las parejas tradicionales; una lógica que se corresponde con la externalización de los servicios, mucho más acentuada en las tareas más rutinarias e intensivas en fuerza de trabajo (García Díez, 2003; Parella, 2003).

A grandes rasgos, el saldo entre trabajo remunerado y no remunerado entre ambos tipos de pareja define las diferencias en el tiempo de ocio. Y este saldo no es cero, sino que la gran renuncia de las parejas de doble ingreso "por no tener tiempo" es el ocio, al cual dedican un tiempo mucho menor que las parejas tradicionales. Concretamente, de las distintas opciones de tiempo libre, aquélla que comporta mayores diferencias es el tiempo empleado en los medios de comunicación, en «ver la televisión».

Si bien prácticamente la totalidad de los miembros de las parejas de doble ingreso declaran trabajar a tiempo completo, la primera característica que distingue hombres de mujeres es, precisamente, la jornada laboral: de lunes a viernes, los hombres dedican a trabajo remunerado o a actividades relacionadas una media de unas nueve horas diarias, dos más que sus parejas, mientras que los fines de semana la diferencia prácticamente es inexistente. No se trata, pues, 
de parejas completamente igualitarias en cuanto a la jornada laboral, aspecto que incide en el resto de tiempos, especialmente en el tiempo empleado en trabajo no remunerado. Si bien en España el tiempo parcial es poco común y más frecuente en mujeres que en hombres (Brullet, 2000; Tobío, 2001; Parella; 2003; Torns y Miguélez, 2007), los datos muestran que las mujeres desarrollan un trabajo que estaría a medio camino entre el tiempo completo y el tiempo parcial.

Por otro lado, y con respecto al trabajo no remunerado, se advierte que las mujeres ocupadas le dedican unas dos horas y media diarias más que sus parejas ocupadas, lo que representa un 119\% más. Tal y como esperábamos, y a pesar de que la diferencia entre ellos y ellas es muy importante, ésta es significativamente menor que la descrita para colectivos más amplios, como son el conjunto de parejas en edad activa o el conjunto de hombres y mujeres que tienen una ocupación laboral: entre los primeros, las mujeres destinan un $202 \%$ más de su tiempo a trabajo no remunerado que los hombres, mientras que entre los segundos la diferencia es de un $155 \%$.

Considerando conjuntamente el tiempo empleado en trabajo remunerado y no remunerado, lo que algunos autores denominan "carga de trabajo» (Ramos, 1990), observamos que, en las parejas de doble ingreso, gran parte de la desigualdad tiene lugar los fines de semana. Así, los días laborables las mujeres trabajan una media hora diaria más que sus cónyuges, mientras que los fines de semana esa diferencia se incrementa hasta prácticamente las dos horas diarias. En este sentido, la menor aportación del hombre al trabajo no remunerado, unánimemente descrita en la bibliografía para el conjunto de parejas (Izquierdo et al., 1988; Ramos, 1990; García Díez, 2003; Aliaga, 2006; entre otros), se podría resumir en las parejas de doble ingreso como que los hombres «ayudan» en las tareas domésticas los días que ella trabaja, mientras que los fines de semana, cuando ninguno de los dos trabaja, la aportación de él a la reproducción es prácticamente inexistente.

El gran perjudicado por esta desigual distribución es el tiempo de ocio de las mujeres (Ramos, 1990; Álvaro, 1996; Raldúa, 2001). Y éste es un cambio importante respecto a lo que sucede en las parejas tradicionales, donde la balanza entre el tiempo de trabajo remunerado del hombre y el de trabajo no remunerado de la mujer termina inclinándose hacia él, de modo que las mujeres disponen de más tiempo libre, de más tiempo para el ocio, que los hombres. Esta conclusión contradice, en parte, la de otros estudios donde, independientemente de la relación con la ocupación, la mujer siempre dispone de menos tiempo libre que el hombre (Gershuny y Sullivan, 1998).

A pesar de que, en las parejas de doble ingreso, la aportación de los hombres al trabajo no remunerado es significativamente mayor que la de sus congéneres de las parejas tradicionales, los tipos de tarea que todos los hombres esquivan son parecidas: independientemente del tipo de pareja, la mayor aportación por parte de ellos (véase gráfico 1) se produce en las tareas menos rutinarias - como las reparaciones en el hogar-, en las que comportan actividades en el exterior - jardinería, sacar a pasear al perro y, en menor medida, gestiones y compras-, 
Gráfico 1. Índice de desigualdad de mujeres y hombres en el tiempo empleado en distintas tareas reproductivas. Parejas de doble ingreso y tradicionales menores de 65 años

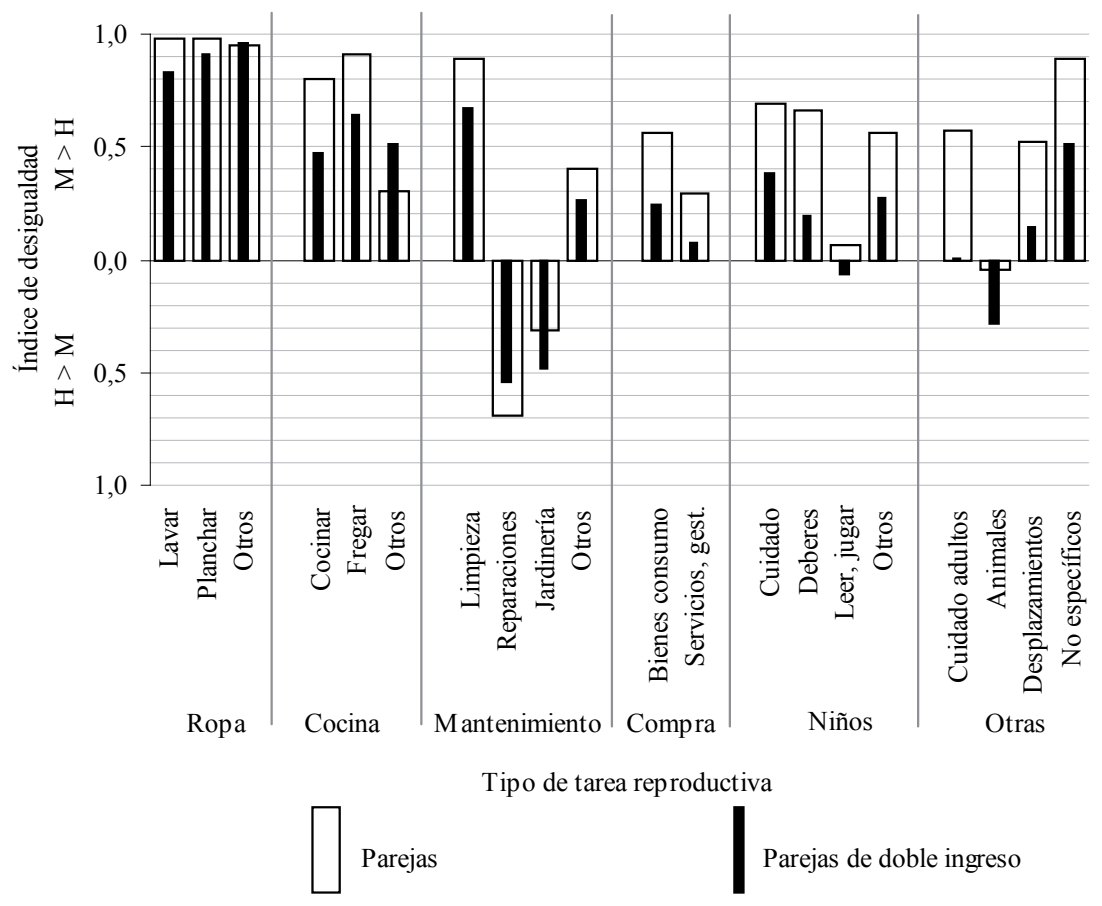

Fuente: elaboración propia a partir de la Encuesta de Empleo del Tiempo 2002-2003.

o en las que comportan un mayor grado de satisfacción o incluso de realización personal. En palabras de Ramos (1990), los varones tienen encomendadas tareas muy periféricas a la economía doméstica y, además, esporádicas.

Para ilustrar mejor la situación en ambos tipos de pareja, se ha dividido el conjunto de tareas en agradables, intermedias y arduas ${ }^{6}$. Pues bien, teniendo en cuenta esta división, en las tareas más agradables, los hombres de las parejas tradicionales sólo «ayudan» a su pareja —ella les dedica el doble de tiempo que él-, mientras que, en las parejas de doble ingreso, los hombres prácticamente las comparten, en cuanto que ellas sólo les dedican un $10 \%$ más que sus parejas. En las tareas intermedias, las mujeres de las parejas tradicionales dedican cinco veces más de tiempo que los hombres, mientras que en las de doble

6. Se han clasificado como arduas las tareas más monótonas, por ejemplo: lavar o planchar la ropa, y la limpieza, ya sea de la vajilla o de los suelos. Como intermedias, se han considerado aquéllas que comportan cierto grado de realización y complejidad, por ejemplo: cocinar, tener cuidado de los niños y también de los adultos. Como agradables, han quedado todas aquellas actividades que se realizan al aire libre y el resto de las que implican a los niños. 
ingreso podría considerarse que los hombres «ayudan», en cuanto emplean aproximadamente la mitad del tiempo que sus parejas. En las tareas más arduas, independientemente del tipo de pareja, los hombres tienen una presencia muy testimonial, pero la diferencia por tipo de pareja es importante: en las tradicionales, las mujeres destinan veintitrés veces más de tiempo que los hombres, mientras que, en las de doble ingreso, la diferencia se reduce a seis veces.

\section{Los condicionantes de las diferencias por género de empleo del tiempo en las parejas de doble ingreso}

Aún habiéndose evaluado otras variables, el modelo más satisfactorio desde el punto de vista de la parsimonia es el generado a partir de cuatro variables independientes: el tipo de hogar, los ingresos de la mujer, la edad de la mujer y el tipo de unión (cohabitación o matrimonio) ${ }^{7}$.

En primer lugar, cabe destacar que el valor estadístico de contraste para la igualdad de medias presenta una significación de 0,000 , por lo que podemos descartar la igualdad de medias en las tres variables dependientes. Al ser posible que las diferencias se den únicamente en una de las tres variables dependientes, se han efectuado modelos para cada variable por separado y se han rechazado en los tres casos la igualdad de medias. Así pues, podemos afirmar que existen diferencias significativas para cada uno de los tiempos analizados.

La primera conclusión a destacar se refiere a las categorías utilizadas como referencia. Para los análisis, se ha tomado como categoría de referencia de cada variable aquella que muestra una mayor igualdad entre hombres y mujeres. Así, las parejas más igualitarias serían las parejas jóvenes (la mujer menor de treinta y cinco años), cohabitantes, sin hijos y donde la mujer dispone de ingresos superiores a los 1.500 euros. Estas parejas son, sin lugar a dudas, las más igualitarias: apenas se observan diferencias en cuanto a los tiempos de trabajo remunerado y no remunerado $(p=0,576$ y $p=0,876$, respectivamente), aunque sí se observa alguna diferencia en el tiempo de ocio, puesto que los hombres cuentan con unos veintiséis minutos diarios más $(p=0,018)$ que sus parejas. Se trata, sin duda, de las parejas más igualitarias en todos los sentidos, de modo que el cambio en cualquiera de las variables comporta un incremento de la desigualdad, sobre todo en el tiempo empleado en trabajo no remunerado.

7. Aunque ha habido otras variables que se han mostrado significativas, se ha excluido su utilización. Así, por ejemplo, no se muestran las que emanan directamente de las condiciones laborales, ya que una de las variables a explicar son las diferencias en el tiempo laboral. También se ha excluido el uso de variables que tengan que ver con la externalización de servicios domésticos, por considerarse variables coyunturales y muy relacionadas con los ingresos. Asimismo, se ha optado por excluir el nivel de estudios de la mujer, dado que mostraba una elevada correlación con los ingresos $(\gamma=0,567)$.

Cabe destacar, además, que, a pesar de que alguna interacción entre estas variables se mostró estadísticamente significativa, desde un punto de vista sociológico, su aportación era muy poco importante, de modo que se ha optado por evaluar el modelo sin interacciones. 
La variable que desempeña un papel más decisivo en la desigualdad, aquella que genera una mayor diferencia entre hombres y mujeres que forman parte de una pareja de doble ingreso, es el ciclo de vida o trayectoria familiar, que viene expresada a partir de la variable «tipo de hogar». Los resultados más interesantes los ofrece el modelo multivariante, donde se comparan hogares de características similares, de modo que permite una primera aproximación a la incidencia que tienen los hijos en la desigualdad ${ }^{8}$.

Así, el primer hijo tiene un impacto muy importante en la desigualdad, lo cual se traduce en el incremento de una hora en las desigualdades en el tiempo de trabajo remunerado y en una hora al trabajo no remunerado. En cambio, el impacto del segundo hijo es mucho menor, ya que las diferencias entre hombres y mujeres apenas sufren alteraciones. El tercer hijo, por otra parte, vuelve a conllevar cambios significativos e importantes, cambios que afectan principalmente a la carga total de trabajo. Así, a grandes rasgos, observamos que la diferencia entre tener un solo hijo o tener tres es que los hombres dedican al trabajo remunerado una media hora más que las mujeres, mientras que ellas dedican al trabajo no remunerado una hora más que ellos, con el consiguiente desequilibrio en la carga de trabajo, una descompensación que va en detrimento del tiempo de ocio de las mujeres $(p=0,045)$.

Además, en menor medida, la variable «ingresos» de la mujer también comporta importantes diferencias entre hombres y mujeres en las parejas de doble ingreso ${ }^{9}$. Estas diferencias van en el mismo sentido que para el conjunto de la población, esto es, que existe una relación inversa entre ingresos y desigualdad (Izquierdo et al., 1988; Ramos, 1990; Larrañaga et al., 2004; González y Jurado, 2009). Ahora bien, que las diferencias sean mayores en los niveles más bajos de ingreso no significa que no existan entre las mujeres que cobran más de 1.500 euros al mes y sus parejas. Sin tener en cuenta el resto de variables, son de poco más de una hora en el trabajo remunerado y de casi dos horas en el no remunerado, con el consiguiente efecto sobre el tiempo de ocio.

En este caso, las diferencias entre el modelo univariante y el multivariante no son muy importantes, de modo que la relativa mayor igualdad entre las mujeres de mayores ingresos y sus parejas debe atribuirse directamente a esta variable. Aunque no se evidencia en nuestro modelo, debe tenerse en cuenta que la literatura muestra que esta menor diferencia se debe más a un decremento de las horas dedicadas por parte de las mujeres, que a un mayor número de horas dedicadas por parte de los varones, de modo que es la externalización de tareas la variable que más explica este cambio, más que el mayor poder de negociación que pueden tener las mujeres con mayores ingresos (Gupta, 2007).

8. El control del resto de variables, pero sobre todo la edad, permite atribuir las diferencias entre una etapa y otra del ciclo de vida directamente a este y no a la edad o generación.

9. Cabe recordar que se han evaluado otras variables, como el nivel de estudios, el nivel de ingresos del hombre, la homogamia educativa y de ingresos, entre otras, y que la variable que mayores diferencias ha generado es el nivel de ingresos de la mujer. Ello no significa que el resto de variables no genere unas diferencias significativas, pero su aportación es mucho menor. 


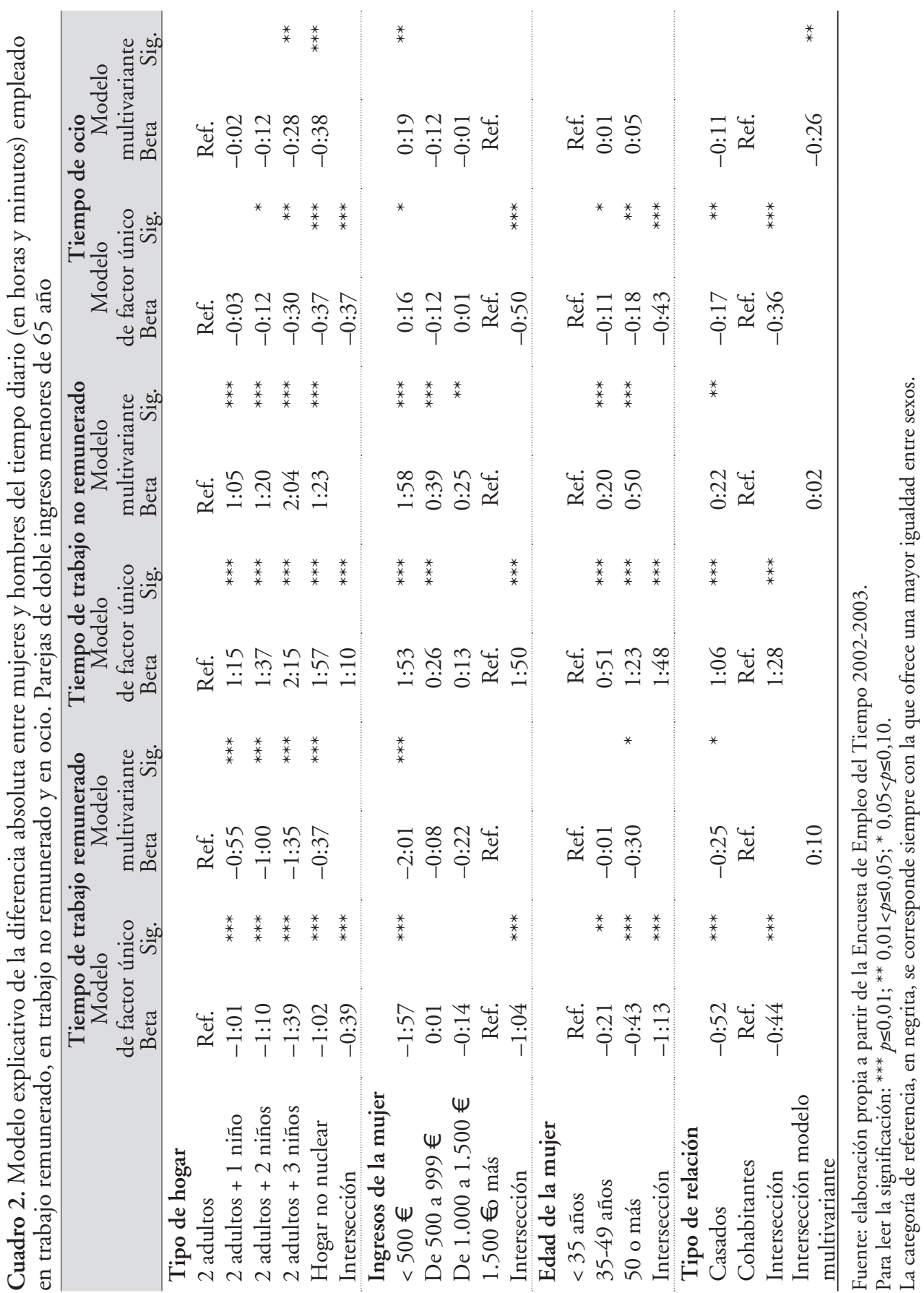


En lo que se refiere al tipo de unión, lo primero que debemos destacar, a diferencia de lo que sucede en las variables anteriores, es que, en el modelo multivariante, los coeficientes se reducen significativamente. Así, analizando solamente el tipo de unión, la conclusión para las parejas de doble ingreso es parecida a la conclusión para el conjunto de la población (García Díez, 2003), es decir, que las parejas cohabitantes son más igualitarias que las parejas casadas. Una desigualdad que en las parejas de doble ingreso se traduce en una hora más desiguales en los tiempos de trabajo remunerado y no remunerado, y en unos quince minutos en el tiempo de ocio. Ahora bien, cuando se introducen el resto de variables, las diferencias son muy inferiores: de 25 minutos en el trabajo remunerado $(p=0,082)$, de 22 minutos en el trabajo no remunerado $(p=0,021)$ y no significativas en el tiempo de ocio $(p=0,199)$. Por ello, debemos concluir que una parte importante de la diferencia observada entre las parejas de matrimonios y cohabitantes debe achacarse a que las segundas son más jóvenes, tienen un mayor nivel de ingresos y forman hogares de solamente dos adultos en una mayor proporción de lo que lo hacen las parejas casadas. Es decir, la mayor equidad entre hombre y mujer mostrada por las parejas cohabitantes se debe más a las características de estas parejas que al efecto bruto de la cohabitación.

Respecto de la edad, debemos destacar, en primer lugar, que, en las parejas de doble ingreso, la relación entre edad y equidad entre hombre y mujer es completamente inversa, de modo que serían las parejas jóvenes las más igualitarias. Y no sólo en el tiempo de trabajo no remunerado, sino que también en los tres tiempos se observa una mayor igualdad, una mayor igualdad que no debe confundirse, ni mucho menos, con una igualdad en términos absolutos. Ahora bien, lo que más destaca de la variable edad no es precisamente esta diferencia, sino que, tal y como sucede con la cohabitación, los coeficientes del modelo univariante difieren de los coeficientes del modelo multivariante. Esta conclusión no es nada esperanzadora de cara a una mayor equidad futura entre hombres y mujeres, y contradice, en parte, la conclusión a la que han llegado otros autores de que las generaciones jóvenes mostraban una mayor equidad. Los resultados muestran que las mujeres más jóvenes se encuentran en una posición de mayor igualdad como consecuencia de sus características, sobre todo como consecuencia de encontrarse muchas de ellas en la fase de pareja sin hijos, una situación que probablemente cambiará a medio plazo y que las igualará a las parejas de mayor edad. Así, si, tal como se ha mostrado, el tipo de hogar es la variable que más influye en el descenso de la igualdad, al tratarse de una variable muy asociada a la edad induce a sospechar que, en las generaciones más recientes, las diferencias entre hombres y mujeres, relativamente pequeñas ahora, incrementarán cuando cambie el tipo de hogar que ahora forman.

\section{Conclusiones}

Es importante recordar, en primer lugar, que estar ocupado o desocupado varía con el tiempo, de modo que algunas parejas que en un momento concreto no 
son de doble ingreso pueden llegar a serlo por el simple cambio en la situación de ocupación de uno de sus miembros a lo largo de la vida, y viceversa. A pesar de esta dinámica, si observamos la foto fija de lo que sucede en España a principios de este siglo, vemos que existen dos tipos de pareja mayoritarios y numéricamente parecidos, pero de características completamente distintas: las parejas tradicionales y las parejas de doble ingreso.

Las primeras se caracterizan por una clara división sexual del trabajo, puesto que son los hombres quienes aportan el sostén económico a la familia, mientras que las mujeres son las responsables del hogar. Se trata de las parejas donde los principios básicos del modelo tradicional apenas han sufrido alteración alguna: aproximadamente un $40 \%$ de las parejas menores de 65 años se encuentran en esta situación. Debido a las características actuales del trabajo remunerado y del trabajo no remunerado, en estas parejas, la carga total de trabajo es superior en los hombres que en las mujeres, de modo que ellas disponen de más tiempo libre que ellos.

Las parejas de doble ingreso, que son aproximadamente otro $40 \%$ del total, sí que han conseguido romper la principal característica del modelo tradicional. A pesar de ello, su situación sigue siendo muy desigual, una desiguadad de la que destacamos tres ejes. En primer lugar, por las diferencias en el tiempo de trabajo remunerado, que muestran que si bien ellos ya no son los únicos que aportan ingresos, siguen siendo los principales. En segundo lugar, por las diferencias en el tiempo de trabajo no remunerado, donde ellas ya no son las únicas cuidadoras, pero sí las principales. Además, la complementariedad de papeles no es simétrica, sino que el papel de ellas en el trabajo remunerado es mucho más importante que la de ellos en el papel de cuidador. Siendo optimistas y evaluando las diferencias como parte de un proceso de cambio de modelo, diremos que, mientras que ellas han hecho los «deberes» y se encuentran cerca del final del camino, a ellos todavía les queda una enorme distancia por recorrer. En términos de tiempo, el principal perjudicado de esta aportación desigual es el ocio, el tiempo libre, porque, a diferencia de lo que sucede con las parejas tradicionales, en las parejas de doble ingreso las mujeres disponen de menos tiempo libre que los hombres. $\mathrm{Y}$, en tercer lugar, porque la incorporación de ellos al trabajo no remunerado no sólo es poco significativa, sino que está completamente desequilibrada, puesto que se han incorporado solamente a las tareas que comportan un mayor grado de realización personal, mientras que, en las más fatigosas, su presencia es completamente secundaria.

$\mathrm{Al}$ analizar estas parejas, se ha comprobado como las diferencias entre hombres y mujeres son menores que si se analizan hombres y mujeres en conjunto, y menores que si se analizan ocupados y ocupadas en general. Ahora bien, no podemos dejar de destacar que las diferencias todavía son muy significativas y lejos de las que se dan en otros lugares de Europa.

Obviamente, no todas las parejas de doble ingreso son iguales. En este sentido, las parejas cohabitantes, que no tienen hijos y en las cuales la mujer es joven y tiene un cierto poder adquisitivo, se encuentran muy cerca de la 
anhelada igualdad. Igualdad que desaparece si no cumple alguna de las cuatro características. En este sentido, se ha puesto de manifiesto que el principal hándicap para la igualdad, aunque no el único, es el nacimiento de los hijos: la desigualdad se incrementa considerablemente con la aparición del primer hijo, el cual para las mujeres significa una pérdida del tiempo productivo, que es substituido por tareas reproductivas, mientras que apenas afecta a los varones. En el extremo, estarían aquellas parejas que tienen tres o más hijos, en las cuales la mujer sacrifica una parte de todos los tiempos - laboral, de ocio, pero incluso personal — para dedicarse al cuidado de los hijos.

En este mismo sentido, si bien es verdad que la igualdad es mayor en las parejas jóvenes, debe tenerse en cuenta que esta igualdad tiene un componente más de edad que de generación, una conclusión que dista mucho de la obtenida en otros estudios. El principal motivo por el cual las mujeres jóvenes se encuentran en una posición de mayor igualdad que el resto es porque todavía no han tenido hijos, una característica que «se cura» con la edad: es muy probable que, dentro de unos años, su comportamiento sea parecido al que observamos actualmente en las mujeres mayores. Así, si bien es cierto que, tanto en España como en otros países, los datos muestran una convergencia significativa entre los sexos a lo largo del tiempo (Gershuny, 1987; García Díez, 2003; MacInnes, 2005), esta convergencia tiene un techo que no parece quebrarse.

Y algo parecido sucede con la cohabitación, de modo que la mayor igualdad observada en las parejas cohabitantes no puede atribuirse a la cohabitación en sí misma, sino simplemente a las circunstancias que rodean a las parejas cohabitantes: en su mayoría más jóvenes que el resto y sin hijos.

Existe un gran desajuste entre el imaginario y lo que sucede en la realidad: mientras que la mayoría de la población opina que la mejor forma de convivencia es aquélla en la cual ambos miembros trabajan fuera del hogar y se reparten las tareas domésticas de forma equitativa (CIS, 2008), en la mitad de las parejas solamente el hombre trabaja fuera del hogar y en la otra mitad el reparto de las tareas no es más que una quimera.

\section{Bibliografía}

Alberdi, Inés (1999). La nueva familia española. Madrid: Santillana.

Aliaga, Christel (2006). «How is the time of women and men distributed in Europe?». Eurostat Statistics in Focus, 4.

Aliaga, Christel y Wingvist, Karin (2003). «How women and men spend their time. Results from 13 European countries?». Eurostat Statistics in focus, 3.

Álvaro Page, Mariano (1996). Los usos del tiempo como indicadores de la discriminación entre géneros. Madrid: Instituto de la Mujer.

BALCELls, Laia (2009). «Analyzing the division of household labor within spanish families». Revista Internacional de Sociología, 67 (1), 83-105.

Beck, Ulrich y Beck-Gernsheim, Elisabeth (1998). El normal caos del amor. Barcelona: El Roure.

BeCKer, Gary (1981). A Treatise on the Family. Cambridge: Harvard University Press. 
Bittman, Michael; England, Paula; Sayer, Liana; Folbre, Nancy y Matheson, George (2003). "When Does Gender Trump Money? Bargaining and Time in Household Work». The American Journal of Sociology, 109 (1), 186-214.

Borràs, Vicent; Torns, Teresa y Moreno, Sara (2007). «Las políticas de conciliación: políticas laborales versus políticas de tiempo». Papers, 83, 83-96.

Bourdieu, Pierre (2000). La dominació masculina. Barcelona: Edicions 62.

Brines, Julie (1994). "Economic Dependency, Gender and the Division of Labour at Home». American Journal of Sociology, 100, 652-688.

Brullet, Cristina (2000). «El debat sobre la conciliació ocupació-família dins el marc de la Unió Europea». Revista Catalana de Sociologia, 12, 155-185.

Callejo, Javier; Prieto, Carlos y Ramos, Ramón (2008). "Cambios generales en el tiempo del trabajo profesional y de cuidados». En: Prieto, Carlos; Ramos, Ramón y Callejo, Javier (coords.). Nuevos tiempos del trabajo: Entre la flexibilidad comptetitiva de las empresas y las relaciones de género. Madrid: Centro de Investigaciones Sociológicas, 5-48.

CIS (2008). Barómetro, junio 2008. Madrid. [http://www.cis.es/cis/opencm/ES/1_ encuestas/estudios/ ver.jsp? estudio=8440]

Dema, Sandra (2005). «Entre la tradición y la modernidad: Las parejas españolas de doble ingreso". Papers, 77, 135-155.

- (2006). Una pareja, dos salarios: El dinero y las relaciones de poder en las parejas de doble ingreso. Madrid: CIS, Siglo XXI.

Durán, M. Ángeles (1986). La jornada interminable. Barcelona: Icaria.

- (2007). El valor del tiempo ¿Cuántas horas te faltan al dia? Madrid: Espasa Calpe.

EAgly, Alice H. (1983). "Gender and Social Influence: A Social Psychological Analysis». American Psychologist, 38, 971-981.

- (1987). Sex Differences in Social Behavior: A Social-role Interpretation. Hillsdale, New Jersey: Erlbaum.

Esping-Andersen, Gosta (1990). Los tres mundos del Estado del bienestar. Valencia: Alfons el Magnànim.

FAGAN, Colette (2003). Working-time preferences and work-life balance in the EU: some policy considerations for enhancing the quality of life. Dublín: European Foundation for the Improvement of Living and Working Conditions.

Franco, Ana y Wingvist, Karin (2002). "Women and men reconciling work and family life». Eurostat Statistics in Focus, 3.

García Díez, Susana (2003). Análisis socioeconómico de la estructura productiva de los hogares: La experiencia española en la última década del siglo XX. Madrid: Consejo Económico y Social.

García Ramón, M. Dolors (1989). «Para no excluir del estudio a la mitad del género humano: un desafío pendiente en geografía humana». Boletín de la Asociación de Geógrafos Españoles, 9, 27-48.

Gasparini, Giovanni et al. (2000). Full-time or part-time work: realities and options. Dublín: European Foundation for the Improvement of Living and Working Conditions.

Gershuny, Jonathan (1987). «¿Estilo de vida, estructura económica y uso del tiempo». REIS, 38, 163-191.

Gershuny, Jonathan y Sullivan, Oriel (1998). "The Sociological Uses of Time-use Diary Analysis». European Sociological Review, 14 (1), 69-85.

Giddens, Anthony (1998). La transformación de la intimidad: Sexualidad, amor y erotismo en las sociedades modernas. Madrid: Cátedra. 
González, Ma José y Jurado-Guerrero, Teresa (2009). “¿Cuándo se implican los hombres en las tareas domésticas?: Un análisis de la Encuesta de Empleo del Tiempo». Panorama Social, 10, 65-81.

Gupta, Sanjiv (2007). "Anatomy, Dependence, or Display?: The Relationship Between Married Women's Earnings and Housework». Journal of Marriage and Family, 69, 399-417.

Hakim, Catherine (2000). Work-Lifestyle Choices in the $21^{\text {st }}$ Century: Preference Theory. Oxford, UK: Oxford University Press.

Ibáñez Pascual, Marta (2008). «La «bolsa común» en las parejas: algunos significados y algunas trampas». Papers, 87, 161-185.

IzQuierdo, Jesusa; Río, Olga del y Rodríguez, Agustín (1988). La desigualdad de las mujeres en el uso del tiempo. Madrid: Instituto de la Mujer.

Larrañaga, Isabel; Arregi, Begoña y Arpal, Jesús (2004). "El trabajo reproductivo o doméstico». Gaceta Sanitaria, 18 (1), 31-37.

LEWIS, Jane (1992). "Gender and the Development of the Welfare Regimes». Journal of European Social Policy, 2 (3), 159-173.

- (2001). "The decline of the Male Breadwinner Model: Implications for work and care». Social Politics, 3 (1), 152-169.

MacInnes, John (2005). «Diez mitos sobre la conciliación de la vida laboral y familiar». Cuadernos de Relaciones Laborales, 23 (1), 35-71.

MÉDA, Dominique (2002). El tiempo de las mujeres: Conciliación entre vida familiar y profesional de hombres y mujeres. Madrid: Narcea.

MerCAdÉ, Anna (2007). «Conciliación entre vida personal y vida laboral». En: López Puig, Anna y Acereda, Amparo (eds.). Entre la familia y el trabajo: Realidades y soluciones para la sociedad actual. Madrid: Narcea, 61-80.

Moreno, Sara (2007). Temps, treball i benestar. Tesis doctoral leída en el Departamento de Sociología de la Universidad Autónoma de Barcelona. Directora: Teresa Torns.

Orloff, Ann Shola (1993). «Gender and the social rights citizenship: The comparative analysis of gender relations and Welfare States». American Sociological Review, 58 (3), 303-328.

- (2006). "From Materialism to "employment for all": state policies to promote women's employment across the affluent democracies». En: Levy, J. (ed.). The State After Statism. Cambridge: Harvard University Press.

Parella, Sònia (2003). Mujer, inmigrante y trabajadora: la triple discriminación. Rubí: Antrophos.

Parella, Sònia y Samper, Sarai (2007). «Factores explicativos de los discursos y estrategias de conciliación del ámbito laboral y familiar de las mujeres inmigradas no comunitarias en España». Papers, 85, 157-175.

Perulli, Ángela (1996). Il tempo da oggetto a risorsa. Milán: FrancoAngeli.

Plantenga, Janneke; Schippers, Joop; Siegers, Jacques (1999). «Towards an equal division of paid and unpaid work: the case of the Netherlands». Journal of European Social Policy, 9 (2), 99-110.

RAldúa, Eduardo (2001). «Comparación internacional de los empleos del tiempo de mujeres y hombres». REIS, 94, 105-126.

Ramos Torres, Ramón (1990). Cronos dividido: Uso del tiempo y desigualdad entre mujeres y hombres en España. Madrid: Instituto de la Mujer.

Sabaté, Ana; Rodríguez, Juana M. y Díaz, M. Ángeles (1995). Mujeres, espacio y sociedad: Hacia una geografía del género. Madrid: Síntesis. 
Tовío, Constanza (2001). «La familia y el empleo: prácticas y discursos de las mujeres». En: Radl Philipp, Rita (ed.). Cuestiones Actuales de Sociología del Género. Madrid: CIS, 129-148.

ToRns, Teresa (2001). «Entre l'atur i el temps parcial: noves maneres per a una vella desigualtat». Revista Catalana de Sociologia, 15, 27-40.

Torns, Teresa; Miguélez, Fausto (coord.) (2007). El temps de treball: balanç d'actuacions a la Unió Europea. [http://www.laboratorideltemps.org/media/ 0000001000/0000001210.pdf]

VALIENTE, Celia (1997). «¿Algo más que "ganadores del pan”?: El papel de los hombres en el ámbito familiar en España (1975-1996)». REIS, 79, 221-263. 